\title{
La arqueología feminista en la normatividad académica
}

\section{Feminist archaeology and academic normativity}

\author{
Sandra Montón SubíAS \\ ICREA/Universitat Pompeu Fabra. Departament d'Humanitats \\ Ramon Trias Fargas 25-27. Barcelona 08005 \\ sandra.monton@upf.edu \\ Sandra Lozano Rubio \\ Universidad Complutense de Madrid. Departamento de Prehistoria \\ Av./Profesor Aranguren s/n. Madrid 28040 \\ sandra.lozano@ghis.ucm.es
}

Recibido: 05-09-2012

Aceptado: 24-10-2012

\begin{abstract}
RESUMen
En sus orígenes, la arqueología feminista desarrolló una crítica a la arqueología dominante en los círculos académicos, o a lo que también se denomina, concisamente, con el anglicismo de mainstream. Esa crítica estaba anclada en la inquietud y el descontento que provocaba experimentar personalmente los sesgos sexistas que afectaban a las mujeres en los diferentes registros del ejercicio de la profesión y estaba dirigida a su modificación. En el presente artículo reflexionamos sobre las razones que dificultan la relación entre feminismo y mainstream, apelando a las incompatibilidades presentes en la lógica interna que guía a cada una de ellas. Asímismo, repasamos brevemente la trayectoria de la arqueología feminista a través de su desarrollo en España y nos detenemos con más detalle en dos aportaciones -la de las actividades de mantenimiento y la de la construcción socio-histórica de la identidad personal. Ambas encarnan, a nuestro modo de ver, un pensamiento feminista cuya lógica es la que provoca desavenencias con el mainstream.
\end{abstract}

Palabras clave: Feminismo. Sexismo. Academia. Actividades de mantenimiento. Identidad.

\begin{abstract}
Initially, feminist archeology developed a critique to dominant or mainstream archeology. Rooted on personal experience of sexism in professional practice it was aimed at transforming it. In this paper, we reflect on the reasons that hinder the relationship between feminist and mainstream archaeology, considering discrepancies present in the internal logic that guides each of them. Likewise, we provide a brief historical overview of the development of feminist archaeology in Spain, and a more detailed account of two approaches: maintenance activities and the socio-historical construction of male and female selves. In our view, both of them embody feminist thinking at odds with mainstream archaeology.
\end{abstract}

KeY words: Feminism. Sexism. Professional practice. Maintenance activities. Identity.

Sumario: Introducción. La arqueología feminista española, brevemente. Los productos del conocimiento científico: valores e intereses en la explicación del pasado. La arqueología feminista y el mainstream en la academia. Con ánimo de continuar. 


\section{Introducción}

La arqueología feminista tiene ya un largo recorrido en EEUU, Inglaterra, Canadá, Australia, Noruega, Suecia, Alemania y España. Sus inicios compartieron en todos estos países una crítica a la arqueología dominante en sus círculos académicos, o a lo que también se denomina, concisamente, con el anglicismo de mainstream. Esa crítica estaba anclada en la inquietud y el descontento que provocaba experimentar personalmente los sesgos sexistas que afectaban a las mujeres en los diferentes registros del ejercicio de la profesión y estaba dirigida a su modificación. Con el paso del tiempo, sin embargo, estas preocupaciones se han ido diversificando. Si para una parte importante de la arqueología feminista los propósitos iniciales -junto con el compromiso político inherente- conservan su vigencia, otras investigadoras han priorizado la integración en el mainstream sobre su cambio transformador, aduciendo un riesgo de segregación.

Precisamente, en este artículo queremos reflexionar sobre las razones de la ardua relación que desde el principio ha existido entre la arqueología feminista y el mainstream. El mainstream, para nosotras, incluye el contenido de la producción científica hegemónica (el discurso y las interpretaciones sobre el pasado) y la norma y los procedimientos -más implícitos que explícitos- que ordenan las relaciones humanas que acompañan a su producción.

Previamente, queremos repasar de modo breve la trayectoria de la arqueología feminista a través de su desarrollo en España. Nos detendremos con más detalle en dos aportaciones, la de las actividades de mantenimiento y la de la construcción socio-histórica de la identidad personal, porque encarnan, a nuestro modo de ver, un pensamiento feminista cuya lógica es la que provoca desavenencias con el mainstream. $\mathrm{Al}$ insistir y reconocer la importancia de la interdependencia y los vínculos sociales, ambas promulgan valores distintos a los de aquellos sobre los que se ha construido la escritura convencional de la historia. Asimismo, nos parece que esta misma circunstancia se da en el ámbito de las relaciones humanas en la práctica arqueológica académica, en la que se recompensan valores y actitudes contrarias a los principios éticos del feminismo. Ello nos servirá, por tanto, para volver al propósito principal del artículo y analizar los fundamentos que rigen la relación entre la arqueología mainstream y la feminista. Sumándonos a trabajos anteriores (por ejemplo, Conkey y Gero 1997; Moser 1996; 2007; Tomášková 2007; Wiley 2007), estamos convencidas de que sigue siendo muy necesario pensar qué significa practicar una arqueología feminista, en todas sus posibles dimensiones. Confiamos que este artículo sea un paso más en ese sentido.

\section{La arqueología feminista española, brevemente}

En la arqueología española, la preocupación explícita por cuestionar el androcentrismo y reflexionar sobre cuestiones vinculadas a las prácticas y experiencias de las mujeres se manifestó a finales de los 80 , aproximadamente una década después que en Noruega y EEUU, donde se habían explicitado por vez primera estas inquietudes. Se publicó entonces el primer artículo sobre el tema (Sanahuja y Picazo 1989), aunque fue un poco más tarde, en 1992, durante la sesión "Arqueología y Mujeres" que tuvo lugar en la Reunión de Arqueología Teórica (RAT) celebrada en Santiago de Compostela, cuando la discusión se desplazó de grupos y personas concretas a un marco académico más general. Al igual que en los países donde ya se habían realizado encuentros similares, varias de las ponentes afirmaron que tanto el ejercicio de la profesión como la interpretación/presentación del pasado participaban de los valores enraizados en la normativa de género contemporánea y reclamaron una cultura disciplinar más equitativa. En retrospectiva, es interesante observar cómo también aquí la reunión se convirtió en un lugar donde compartir vivencias, proporcionó referentes para otras mujeres arqueólogas que habían experimentado el sexismo en la profesión e impulsó el interés por el tema. Desde entonces, los estudios feministas se han convertido en uno de los ámbitos más dinámicos de la disciplina en nuestro país. Un ámbito con entidad propia en el contexto global de la arqueología feminista y en el contexto particular de la discusión teórico-metodológica peninsular.

Sin embargo, al igual que ocurre en la escena internacional, se ha minimizado o comprendido de modo insuficiente la contribución de la arqueológica feminista, pues básicamente se la ha confinado al terreno de la socio-política, sobre todo por lo que concierne a la reparación de la doble discriminación de las mujeres en el pasado y en el presente (cf. Montón Subías 2010b: 3-4; Montón Subías y Meyer en prensa). Sin minimizar la importancia de sus políticas de representación, nos parece también muy importante destacar que la arqueología feminista ha aportado a la disciplina autocrítica y reflexión, interpretaciones históricas alternativas y el desarrollo de nuevos campos de investigación. En 1970, por ejemplo, cuando la arqueología feminista dio sus primeros pasos en Noruega y EEUU, en plena consolidación del procesualismo, fue sobresaliente reconocer que las interpretaciones dominantes sobre el pasado reflejaban intereses y valores particulares de quienes las promulgaban, desfavorecían a las mujeres y legitimaban la ideología de género hegemónica. Así, desde el principio, la crítica 
al androcentrismo disciplinar puso en tela de juicio la epistemología tradicional al reconocer el carácter situado de la investigación y de sus productos (ver, por ejemplo, la introducción de Bertelsen et al. 1987- seminario que tuvo lugar en 1979); algo que, sin embargo, ha pasado al imaginario arqueológico como un logro de la arqueología posprocesual iniciada en Cambridge durante los 80 (ver también Hernando, en este volumen).

La introducción del feminismo en la arqueología española también ha propiciado la reflexión teórica, la revisión crítica de las prácticas y narrativas arqueológicas, la formulación de nuevas hipótesis para interpretar o reinterpretar diferentes conjuntos del registro arqueológico peninsular, el desarrollo de temas inéditos hasta el momento y de nuevas categorías de análisis para entender la dinámica histórica de los grupos del pasado. Así, se han escrito estudios específicos sobre el sesgo sexista del discurso arqueológico y del lenguaje -verbal y no-verbalcon que se expresa (Argelés et al. 1991; González Marcén 2008a; Hornos y Rísquez 2005; Lozano 2011a; Querol 2000; 2008; Querol y Hornos 2011; Sanahuja 1991; Soler 2008); se han reevaluado los conceptos de tiempo, espacio e identidad personal (González Marcén 2008b; Hernando 2000; 2002; 2012; Montón Subías 2000; Pallarés 2000) y el registro funerario (Aranda et al. 2009b; Castro et al. 1993-94; Izquierdo 2007; Montón Subías 2007; 2010a; 2011; Prados 2010; Rísquez y García Luque 2007; Rísquez et al. 2010; Sanahuja 2006); se ha visibilizado el trabajo y las aportaciones de las mujeres en el pasado, olvidadas o estereotipadas en las narrativas tradicionales (Colomer 2005; Delgado y Ferrer 2007; Fernández 2000; Orozco 2005; Prados 2007; Sánchez Romero 2000; Sánchez Romero y Moreno 2005), y en la profesión (Cárdaba et al. 1998; Díaz-Andreu y Sanz Gallego 1994); y se ha cuestionado, aunque de modo todavía incipiente, los roles y modelos de masculinidad proyectados desde el presente (Aranda et al. 2009a). No han faltado tampoco reflexiones y/o síntesis generales sobre la arqueología de género, feminista y de las mujeres (Colomer et al. 1999; Cruz Berrocal 2009; Díaz-Andreu 2005; Díaz-Andreu y Montón Subías 2012; González Marcén 2006; Lozano 2011b; Montón Subías en prensa; Montón Subías y Meyer, en prensa; Sánchez Liranzo 2008; Sánchez Romero 2009; Vila 2011; Zarzalejos 2008), análisis del género desde la etnoarqueología (Hernando 2010; Hernando et al. 2011), o estudios sobre género y arte (Escoriza 2002; Hernández 2005; Lozano 2008; Masvidal y Picazo 2005).

Igual que en los países pioneros, todas estas cuestiones empezaron introduciéndose en la universidad desde posturas feministas. Fue diferente, sin embargo, el contexto teórico-metodológico que las recibió y el paradigma que las auspició, pues en España predominaba el historicismo cultural y la descripción de materiales sobre el debate teórico. Esta manera de hacer arqueología fue cuestionada principalmente desde el marxismo, bien informado, no obstante, por la metodología procesual tan en boga en el mundo angloparlante. Esta singular presencia de las tesis marxistas en las universidades españolas ha de entenderse en el marco históricopolítico inmediatamente posterior a la dictadura franquista (véase Vicent 1994). Así, las primeras arqueólogas con inquietudes feministas partían de una fuerte base marxista que las llevó a cuestionar los análisis de género procedentes del mundo anglosajón. Desconfiaban de lo que consideraban un tibio compromiso político y un escaso interés por la ortodoxia materialista. Así se explica la preferencia inicial por el término sexo y la desconfianza hacia la categoría género (ver, como un ejemplo, Colomer et al. 1993: 5). Solamente con posterioridad, ante el empuje imparable de la producción anglófona y la influencia de otras disciplinas, como la historia o la antropología, la arqueología del género en sentido estricto -la que incorpora el género como categoría analítica-ingresó también en la academia española. Comenzaron así otras arqueologías feministas, que, a partir de entonces, convivieron con la marxista inicial. El deseo explícito de diferenciarse de las arqueologías de género continúa presente en algunas de las propuestas de tradición marxista actuales (Escoriza y Sanahuja 2005; Pérez 2011; Sanahuja 2002; Vila 2011 y, para repasar algunos de los aportes más significativos del feminismo marxista en España, Vargas 2004). Por ello, en España, al margen de que haya arqueologías de género feministas y no feministas también hay arqueologías feministas que pueden ser o no de género (Montón Subías, en prensa).

\section{Los productos del conocimiento científico: valores e intereses en la explicación del pasado}

Ante la ya larga trayectoria del feminismo en el mundo académico, debería quedar claro que uno de los principales objetivos de la arqueología feminista, al estar comprometida políticamente con el final del patriarcado, consiste en promover un cambio de cultura disciplinar que acabe con sus sesgos sexistas y -sobre todo tras la aportación queer- también heterosexistas. Ello ha incluido e incluye reparar la invisibilidad de las mujeres en la explicación del pasado, sin duda una estrategia muy seguida $-\mathrm{y}$ muy lógica- en su primera andadura, tras décadas de olvido y/o mera reproducción de estereotipos. 
Sus propósitos, no obstante, desde siempre han sido más amplios, profundos y espinosos (ver, por ejemplo, Conkey y Spector 1984).

Como parte de ese cambio de cultura disciplinar, la arqueología feminista invita a pensar sobre los valores que deben incorporarse a la escritura de la historia y, en su medida, a contribuir a la construcción de una nueva racionalidad pública (Montón Subías, en prensa). No se trata únicamente de integrar nuevos agentes en forma de mujeres, niños, ancianos, terceros géneros, etc., para completar las explicaciones tradicionales -algo bastante aceptado desde la academia normativa-, sino de escudriñar los valores e intereses sobre los que y con los que se construye el discurso teórico mainstream y des-velar que su lógica más profunda es la lógica del orden patriarcal. En este sentido, al igual que el feminismo político se ve fuertemente contestado cuando señala las injusticias más estructurales, la arqueología feminista ve frenados sus objetivos más ambiciosos, que son duramente contestados o ignorados por el mainstream al sentirse cuestionado en los cimientos androcéntricos que lo sostienen.

Precisamente, en esa labor de introspección académica, las teóricas y teóricos del feminismo han demostrado, en diferentes campos de las Ciencias Sociales y las Humanidades, que el androcentrismo ha influido negativamente en el conocimiento científico al sesgarlo de raíz (Intemann 2010: 792); al producir relatos que únicamente enaltecen valores, actitudes y capacidades asociadas a la llamada "masculinidad hegemónica" (Connell y Messerschmidt 2005): la individualidad, el poder, la razón, el autocontrol, la violencia -en tanto que ejercicio para obtener, ejercer o recuperar el poder-, el cambio, el crecimiento tecnológico o la competitividad. El androcentrismo está basado en una lógica que se asienta sobre la ocultación y el rechazo de aquellos otros valores, actitudes y capacidades considerados privativos de las mujeres (cf. Seidler 2000: 18) y crea un cisma abisal entre razón (cultura) y emoción (naturaleza) (Arneil 1999; Beauvoir 1949; Ortner 1974; MacCormack y Strathern1980; Rivera 1994: 207; Shiva 1998; Hernando, en este volumen). No es muy difícil reconocer que esa es también la tónica en que se alinean los "grandes" temas de la investigación arqueológica, con independencia, como acertadamente ha señalado Hernando (en prensa), del paradigma científico desde el que se emprende (ver también Escoriza y Sanahuja 2005: 111). En ese trabajo de reflexión, además, las mujeres y, entre ellas, las arqueólogas feministas, por su experiencia situada, por no haber formado parte nunca de la masculinidad dominante, parten con ventaja. Es lo que numerosas pensadoras feministas -elaborando conceptos del marxismo (cf. Harding 1986:
26; Potter 2006: 135; Tanesini 1999: 139-140)- han denominado el privilegio epistémico de las mujeres como grupo subordinado, más expuestas a observar la parcialidad del conocimiento que las excluye (Hartsock 1983; Rose 1983; Smith 1988; Collins 1990; Wylie 2003). Es ésta una condición particular -la de situarse fuera de la masculinidad dominanteen la que también se puede estar desde el indigenismo (Atalay 2012; Watkins 2000), las etnicidades subalternas y discriminadas (Blakey 1983; Franklin 2001) o la sexualidad no normativa (Dowson 1998; Voss 2008).

Desde la arqueología peninsular, hace tiempo que se viene insistiendo en que la historia puede y necesita escribirse de otra manera. Nos detendremos precisamente ahora en dos de estas propuestas: la de las actividades de mantenimiento y la de la construcción socio-histórica de la identidad personal. Ambas proponen maneras diferentes de entender a los grupos humanos, al destacar la valía del vínculo y la conexión social, la capacidad afectiva y la práctica de la relación y del cuidado como pilares fundamentales de la vida social. Al incidir en ello, reclaman la importancia de la relacionalidad, la interdependencia, la estabilidad, la perdurabilidad, la permanencia y la recurrencia en el transcurso de la historia, precisamente los aspectos olvidados en la narrativa tradicional al considerarse antagónicos a la masculinidad hegemónica desde la que se han escrito.

El concepto de actividades de mantenimiento se originó a principios de los años 90 de la mano de un grupo de arqueólogas feministas de la UAB, dentro del énfasis dado por el feminismo de inspiración marxista al análisis de las formas de trabajo y las condiciones materiales de la existencia de las mujeres. La propuesta proponía profundizar en el examen de una determinada esfera de la producción y reproducción social, una esfera que había sido olvidada en la narrativa histórica tradicional (incluida la marxista). Muy pronto se unieron otras investigadoras, que vieron en este concepto una herramienta útil para visibilizar y evaluar las experiencias de las mujeres en el pasado. Actualmente, esta propuesta continua enriqueciéndose con las aportaciones de diferentes investigaciones dentro y fuera de España (ver, por ejemplo, Montón Subías y Sánchez-Romero 2008).

Tras más de dos décadas de desarrollo, la literatura sobre las actividades de mantenimiento incluye reflexiones teóricas generales sobre las implicaciones y dimensiones del concepto (Picazo, 1997; González Marcén y Picazo, 2005; González Marcén et al., 2008; Montón Subías, 2000), estudios específicos sobre actividades concretas como la alimentación (Colomer, 1996; Montón Subías, 2002), 
el cuidado (Alarcón, 2007; Montón Subías, 2010c; Sánchez Romero, 2007), la socialización de niños y niñas (Sánchez Romero 2007; 2008) y análisis de contextos arqueológicos específicos (Alarcón et al., 2008; de Pedro, 2006). Además, las actividades de mantenimiento se han desvelado como una herramienta de gran potencial para evaluar dinámicas sociales de larga duración y evaluar desde una óptica diferente los periodos considerados de transición histórica (Colomer et al., 1998; Curià y Picazo, 2000; González Marcén et al., 2007; Sánchez Romero y Aranda, 2005).

Se entiende por actividades de mantenimiento toda una serie de trabajos básicos y necesarios para garantizar la estabilidad y la reproducción de la vida y sus formas en cualquier grupo humano. Estas actividades y cómo se organizan varían en función de la cultura, pero grosso modo comprenden todas las relacionadas con la recogida, procesamiento y cocinado de alimentos, la manufactura textil básica, la higiene y la salud públicas, la atención y el cuidado al otro y la socialización de los miembros infantiles. La variación cultural que pueden presentar no desdibuja, sin embargo, el papel estructural que desempeñan. Se desarrollan en la escala de la cotidianeidad y son indisolubles del entramado relacional que crean y en el que se despliegan. En este sentido, son las garantes de los vínculos básicos que mantienen la cohesión grupal. Es necesario aclarar que algunas de estas actividades, como por ejemplo la cocina o la producción textil, con una presencia material evidente en el registro arqueológico, han sido atendidas con mucha frecuencia en arqueología, incluso sin que ello implicara necesariamente una aproximación feminista o comprometida con el análisis de género. La novedad de englobarlas bajo una denominación común reside en destacar la función colectiva que comparten todas ellas y que, de otra manera, queda desdibujada. Su unión conceptual proporciona, además, una base clara para rastrear el desarrollo de los valores sociales comunes que encarnan. La propuesta, pues, subraya la función estructural de estas actividades, que, desde la cotidianeidad, garantizan la resiliencia social (Colomer et al. 1999: 54) al asegurar que los cambios se absorban en nuevos patrones de repetición y recurrencia que devuelven la estabilidad básica y permiten la supervivencia del grupo (González Marcén et al. 2008: 4). Analizar la cotidianeidad en periodos históricos de larga duración, sus formas de permanencia y transformación, permite entender mejor la articulación de las diferentes variables -también las consideradas macro- que configuran el tejido social, así como sus cambios y modificaciones.

Asimismo, la propuesta subraya la importancia de la relacionalidad y la interdependencia que es- tas actividades alimentan -consideradas hasta hace poco exclusivas de la identidad femenina-, en línea con lo que proponen autoras agrupadas en torno a la "ética del cuidado", como Nancy Chodorov, Susan Leigh Star o Carol Gilligan, autoras marxistas no ortodoxas como Anna Jónasdóttir y autoras vinculadas al feminismo de la diferencia sexual conectado con la Librería de Milán como Luisa Muraro y Adriana Cavarero. Esta empresa se comparte con otra de las aportaciones más originales de la arqueología feminista en España: la que propone explicar las razones históricas de la desigualdad de género en el mundo occidental profundizando en el estudio de los mecanismos mediante los que se construye la identidad personal y, más concretamente, en el análisis de la interacción entre la identidad relacional y la individualizada.

Esta línea de investigación, que surgió en Madrid a finales de los 1990, arrancó de un punto de partida diferente a la de la mayor parte de los estudios feministas, ya que no fijó inicialmente la atención en las mujeres, sino en el modo en que los seres humanos construyen su identidad (Hernando $2002 ; 2005)$. Sobre la base de sus propios trabajos etnoarqueológicos y de los estudios que en filosofía, psicología, sociología y antropología ya habían profundizado en las diferencias entre la identidad relacional e individualizada, Hernando propuso que individualidad y relacionalidad constituyen "paquetes" de rasgos que se combinan de forma dinámica y compleja dentro de una misma persona, dependiendo de su posición de poder o control en el grupo social (Hernando 2012). Partiendo de estos presupuestos, la autora analizó la genealogía de las identidades de género contemporáneas en el mundo occidental, concluyendo que inicialmente todos los hombres y mujeres de las sociedades humanas tendrían rasgos identitarios relacionales (semejantes, en términos estructurales, a los que caracterizan a los cazadores-recolectores actuales; ver Hernando, en este volumen). Una identidad, la relacional, que también se despliega en el ejercicio de las actividades de mantenimiento, que, de acuerdo a la anterior propuesta, habrían dominado los inicios de la vida en grupo. Ambas explicaciones, por lo tanto, escapan de esencialismos vinculados al sexo.

Según Hernando (íbidem; este volumen), los hombres -especialmente aquellos conectados con esferas de poder- habrían ido desarrollando lenta y gradualmente la individualidad, al tiempo que las mujeres habrían mantenido la identidad relacional (que antes caracterizaba a todos los miembros del grupo y que es necesaria para garantizar la cohesión social y afectiva de los grupos humanos). A partir de la modernidad, las mujeres también habrían desarrollado los rasgos de individualidad, pero en virtud 
de sus diferentes trayectorias históricas, existiría una diferencia entre la que actualmente caracteriza a hombres y mujeres, ya que los hombres se habrían asegurado participar de la imprescindible identidad relacional a través de relaciones complementarias y desiguales con mujeres especializadas en ese tipo de identidad, mientras que las mujeres de la modernidad han tenido que encarnar ellas mismas ambos tipos de identidad (la relacional y la individualizada) porque los hombres no han sido entrenados históricamente para servirles a ellas de "complemento emocional" . Ya hemos indicado antes que la identidad individualizada se asocia en nuestra normativa de género a la masculinidad hegemónica. Es cierto que desde diversos lugares se viene insistiendo en que la masculinidad hegemónica es una expectativa socialmente construida y, por lo tanto, un mito, una ficción, una fantasía, un modelo ideal (cf. Blazina 2003; Connell, 1995; Easthope 1990; Guasch 2006; Pleck 1983). Hernando, además, ha estudiado cómo la forma de individualidad que la caracteriza se sustenta históricamente sobre los valores -los de la relacionalidad- que niega y enmascara (Hernando 2012, este volumen, y ver también sobre este punto Seidler 2000: 18). El discurso arqueológico de la Modernidad, al defender un reemplazo progresivo de la identidad relacional por la individualizada $-y$ de la emoción por la razón- desde la prehistoria no ha hecho más que crear una ficción, proyectando al pasado la lógica presente que se desea justificar.

Ninguna de las anteriores propuestas se propone invertir los esquemas para glorificar los valores asociados a la relacionalidad como opuestos a los de la individualidad, sino reivindicar el necesario reconocimiento de unos valores y capacidades que, sostenidos tradicionalmente por las mujeres en nuestro particular devenir histórico, se han presentado como antagónicos sin serlo, unos valores y capacidades sin los cuales la vida humana resulta insostenible (Hernando 2012; Montón Subías 2010c: 28).

\section{La arqueología feminista y el mainstream en la academia}

La academia y la universidad - una de sus instituciones principales- configuran un espacio público por antonomasia; un espacio público desde donde se nombra el mundo, desde donde "se pone en palabras lo que es la ciencia, lo que es la sociedad, lo que son las artes, la política, la historia, el pensamiento, la salud, la justicia, las relaciones humanas, etc." (Rivera 2005:1161) en un marco relacional que, "hecho a imagen de los hombres" (Seidler 2000: 1), tiene que ver, principalmente, con el poder y la jerarquía. Un marco donde, por lo tanto -de manera análoga a lo que comentábamos antes respecto al contenido del conocimiento arqueológico-, sobre todo recompensa valores, actitudes y comportamientos que tienen que ver con la masculinidad normativa. Valores, actitudes y comportamientos que dominan, efectivamente, las relaciones que se establecen entre el profesorado y el alumnado, entre los investigadores e investigadoras consolidados y quiénes están en proceso de hacerlo $\mathrm{y}$ entre los propios investigadores e investigadoras consolidados en el esfuerzo por obtener recursos con los que multiplicar poder. Las relaciones jerárquicas y asimétricas que a menudo rigen la dirección de becas y tesis doctorales, el liderazgo de proyectos de investigación o simplemente la firma -y su orden- de las publicaciones científicas son algunos ejemplos. Llevados a sus últimas consecuencias, en su forma más exacerbada, se hallan detrás de las malas prácticas científicas, sin duda la cara más fea de la ciencia: endogamia, servilismo, clientelismo, autorías "honoríficas", falsificación de datos, violencia psicológica y un etcétera más largo que puede consultarse en http://www.corruptio.com/. Algo que se denuncia cada vez más en publicaciones académicas, también en España (Cruz Berrocal en prensa; de Simone 2000; González Ruibal 2011; Penalva 2011; Peñasco 2005; Piñuel 2005; Vazquez-Cupeiro y Elston 2006), y que lleva tiempo expresándose en el mundo de la narrativa, a través, por ejemplo, de la denominada "ciencia en la ficción" (Djerassi 1989; 1998).

El feminismo, en tanto que ejercicio político, se opone a cualquier sistema que genere explotación y dominio (Collins 1990: 37-38), y, por lo tanto, a unas prácticas científicas que se apoyan en la acumulación de poder y se alimentan de desigualdad. En este sentido, desafortunadamente, sus repercusiones en la academia continúan siendo extremadamente limitadas, a pesar del éxito relativo que puedan haber tenido sus políticas de representación, dobles en el caso de la arqueología al reivindicar la incorporación de las mujeres a la práctica de la profesión y a los relatos sobre el pasado. Es precisamente aquí donde reside la contradicción principal entre el feminismo y el mainstream, donde su cohabitación se vuelve enrevesada, tensa, ardua y difícil, al regirse ambos por criterios y reglas de procedimiento incompatibles por principio. De ahí, también, el nudo que sienten algunas investigadoras -y también algunos investigadores-al seguir unas pautas que se contradicen con sus convicciones (cf. Caballero 2010, Arnaus 2010). En nuestro caso concreto, la arqueología feminista, este nudo se hace incluso más intenso al evidenciarse la disfunción entre aquellos valores que se quieren rescatar en el relato del pasado y los que se potencian en la práctica cotidiana de la investigación, precisamente los que se han tachado de androcéntricos en las interpretaciones históricas. 
Entre algunos sectores de nuestra disciplina, se ha generado la falsa impresión -sobre todo a partir de la relación superficial y malentendida que se ha establecido en ocasiones entre arqueología feminista y arqueología de género (por ejemplo, Renfrew y Bahn 2012: 225-228)- de que el feminismo ya ha ocupado su espacio en el mainstream y, además, de forma muy visible (ver con todo la crítica de Fernández 2006: 149). Pretender que el feminismo pueda alterar el mainstream de forma inadvertida, sin provocar rechazo ni molestias resulta ilusorio porque, asumido en todas sus consecuencias, alteraría cada aspecto de la práctica científica. De hecho, en ocasiones, ha ocurrido justo lo contrario, y ha sido el mainstream el que ha modificado algunos desarrollos de la arqueología feminista. Los estudios de género nos parecen un ejemplo claro, pues aunque fueron feministas quienes los incorporaron a nuestra disciplina (Conkey y Spector 1984), el anhelo de mainstream a cualquier precio, el temor al ostracismo (o a ser etiquetadas de tendenciosas y poco científicas) y la suscripción oportunista se han combinado para desactivar, en algunos casos, su crítica feminista inicial (cf. Engelstad 1999; 2007; Montón Subías en prensa). Algo, por otra parte, frecuente en la era posmoderna, donde la inclusión de la diferencia integra en realidad solo la apariencia de diferencia y neutraliza la verdadera diferencia (Benavides 2005).

De modo que, aunque la presencia de los estudios feministas y de género resulta ya incontestable en nuestra disciplina desde hace tiempo, paradójicamente ello no ha supuesto una modificación sustancial de las dinámicas de poder androcéntricas. Es cierto, no obstante, que algunas arqueólogas han criticado el sexismo de una de nuestras prácticas más definitorias, el trabajo de campo (Gero 1996 -aunque ver contestación de Politis 2001; Moser 1996; 2007; Ruiz 2008), y sugerido algunas pautas para modificarlo (Conkey y Gero 1997; Tomaskova 2007). De hecho, en el RAT de Santiago que mencionábamos antes, una de las comunicaciones -que nunca se publicó- iba en esta misma dirección al preguntarse si todas éramos hombres en arqueología (Colomer et al. 1992). Personalmente, estamos convencidas de la necesidad de continuar meditando sobre la concreción e implicaciones de nuestro compromiso político en la modificación de la cultura académica, sobre el marco relacional que abraza la generación del conocimiento arqueológico, sobre la academia como espacio público de poder. La arqueología feminista es también un estado de alerta sobre las dinámicas de poder que dominan la disciplina, un estado de crítica y auto-crítica constante (Conkey 2005; Tomaskova 2007: 3; Wylie 2007). De otro modo corremos el riesgo de modificar el discurso arqueológico - la cara más visible, la que se publica-, pero perpetuar una estructura androcén- trica en los engranajes de su producción (cf. Montón Subías, en prensa).

Afortunadamente, el patriarcado nunca ha ocupado toda la experiencia humana (Rivera 2000: 105), tampoco en la Academia; si no, sería imposible cuestionar su lógica, ni plantear alternativas. El patriarcado tiene también resquicios para que la crítica tenga lugar, en la línea de lo que expresaron sociólogos como Giddens (1984); la estructura limita pero también posibilita el cambio. En ese sentido, diferentes investigadoras e investigadores están proponiendo ya alternativas para impregnar la práctica científica de los valores que predica el feminismo. En nuestra disciplina, Alison Wylie (2007: 212), por ejemplo, invita a trabajar desde una ética diferente, que elimine toda práctica de explotación y dominación e implemente relaciones igualitarias que se contrapongan a la práctica académica normativa actual, vertical y jerárquica. En nuestro país, desde distintas disciplinas, varias voces (Arnaus y Piussi 2010; Comins 2009; Guasch 2006; Montón Subías 2010c; Salazar 2012) invitan a incorporar los valores de la cotidianeidad y la ética del cuidado (Gilligan 1982) a la esfera pública de poder. Valores que, estrechamente asociados a las tareas tradicionales de las mujeres, tienen que ver con la intersubjetividad, el reconocimiento, la solidaridad y la ternura (Comins 2009). Investigadoras del pensamiento de la diferencia sexual vinculadas a los centros de investigación Duoda (Universidad de Barcelona) y Diotima (Universidad de Verona), además, han reflexionado muy específicamente sobre la universidad. Reconocen la paradoja que supone que la universidad sea -hoy más que nunca- numéricamente femenina pero cultural y políticamente masculina y proponen una alternativa basada en la incorporación de lo que ellas denominan el "orden simbólico de la madre" (Arnaus y Piussi 2010; Rivera 2010: 57); una alternativa que, "conservando lo que de valioso tiene la tradición masculina" (Rivera 2010: 57) introduzca en la universidad prácticas de relación diferentes, que rompan con las asimetrías y sustituyan el poder por autoridad en los diferentes ámbitos de la gestión, la docencia y la investigación (Alvárez Galván 2010; Arnaus y Piussi 2010).

\section{Con ánimo de continuar}

En las páginas anteriores, hemos planteado los motivos que, desde nuestro punto de vista, complican la convivencia entre la arqueología feminista y la normatividad arqueológica. El mainstream se resiste -mediante la reacción o la indiferencia- a incorporar discursos del pasado articulados en torno a valores e intereses diferentes a los que le caracterizan, a la vez que las investigadoras e investigadores 
feministas se ven inmersas en unas prácticas profesionales que se contradicen con los principios que inspiran sus trabajos científicos. Creemos que es importante tomar conciencia de esta circunstancia, aunque resulte extremadamente difícil modificarla. Sabemos lo complicado que resulta construir una carrera académica practicando dinámicas distintas a las que dominan el "campo científico" (sensu Bourdieu, 1975), que sentimos cada vez más acelerado, apresurado, agobiado de trámites y requisitos que convierten en lujo no solo el tiempo de la relación y la vida (de Vita 2010: 84), sino el de la reflexión y sosiego del que, paradójicamente, se nutre la buena investigación. Investigadoras que llevan más tiempo recapacitando sobre este dilema, se reconocen en ocasiones desanimadas, con la sensación o la duda de haber creado lugares paralelos dentro de la universidad, en vez de haber transformado la tradicional institución masculina (ver por ejemplo, Rivera 2010: 51; Zamboni 2010: 74). Convenimos con ellas (y con otros y otras) en la necesidad de continuar trabajando desde la práctica cotidiana para ampliar los márge- nes que hospedan esas otras maneras de actuar; unos márgenes que permitan practicar con cierta eficacia unas relaciones sociales profesionales más acordes con los principios del feminismo. El potencial de los discursos alternativos sobre el pasado se quedará a medias si quienes lo proponen no llevan su reflexión sobre los seres humanos del pasado a las prácticas de los seres humanos del presente. Ello, además de contrarrestar la tradición masculina patriarcal, redundará en beneficio de una ciencia más democrática (sensu Longino 1990). Se romperá así desde dos frentes en nuestro caso, la interpretación del pasado y las relaciones humanas en el presente, con los patrones socio-culturales del patriarcado.

Personalmente, no queremos salir de los márgenes, sino convertir el margen en mainstream "de nuevo, como hizo con el patriarcado y su política sexual el feminismo del siglo XX, con una revolución sin sangre, o sea, con una o muchas revoluciones simbólicas que desvinculen el conocimiento del poder, que lo saquen (el poder) de la vida universitaria" (Rivera 2010: 68).

\section{Agradecimientos}

Queremos expresar nuestro más sincero agradecimiento a los editores de este monográfico de Complutum por habernos invitado a participar en él. También queremos agradecer la ayuda de colegas, amigas y amigos que leyeron un primer borrador del texto y nos ofrecieron comentarios e información para incluir en el texto. Nuestro más sincero agradecimiento, por lo tanto, a Albert Presas, Almudena Hernando, Apen Ruiz y María Cruz Berrocal.

\section{REFERENCIAS BibLIOGRÁFICAS}

Alarcón, E. (2007): Las prácticas de cuidados en las sociedades prehistóricas: la cultura argárica. Arqueología y Territorio, 3: 233-249.

Alarcón, E.; Sánchez Romero, M.; Moreno, A.; Contreras, F.; Arboledas, L. (2008): Las actividades de mantenimiento en los contextos fortificados de Peñalosa. Cuadernos de Prehistoria y Arqueología de la Universidad de Granada, 18: 265-296.

Álvarez Galván, F. M. (2010): Doble sí, doble saber: maternidad y universidad. En Arnaus y Piusi 2010: 145-154.

Aranda, G.; Montón Subías, S.; JimÉnez-Brobeil, S. (2009a): Conflicting evidence? Weapons and skeletons in the Bronze Age of south-east Iberia. Antiquity, 83 (322): 1038-1051.

Aranda, G.; Montón Subías, S.; SÁnchez-Romero, M.; Alarcón, E. (2009b): Death and everyday life. The Argaric societies from Southeast Iberia. Journal of Social Archaeology, 9: 139-162.

Argelés, T.; Piqué, R.; Vila, A. (1991): La importancia de llamarse hombre en prehistoria. Revista de Arqueologia, 121: 6-9.

Arnaus, R. (2010): El sentido político de la creación femenina. En Arnaus y Piusi 2010: 155-184.

Arnaus, R.; Piussi, A.M. (eds.) (2010): La Universidad Fértil. Mujeres y hombres, una apuesta política. Octaedro, Barcelona.

ArneIL, B. (1999): Politics and Feminism. Blackwell, Oxford.

Atalay, S. (2012) Community Based Archaeology: Research with, by and for Indigenous and Local Communities. University of California Press.

BeauvorR, S De. (1949): Le Deuxième Sexe. Gallimard, Paris. 
Benavides, O. (2005): Los ritos de autenticidad: indígenas, pasado y el estado ecuatoriano. Arqueología Suramericana, 1(1): 5-48.

Bertelsen, R.; Lillehammer, A.; NÆss, J. R. (eds.) (1987): Were they all men? An examination of sex roles in prehistoric society. Arkeologist museum i Stavenger, Stavenger.

Blakey, M. (1983): Socio-political bias and ideological production in historical archaeology. The Socio-Politics of Archaeology (J.M. Gero; D.M. Lacy y M.L. Blakey, eds.), Research Report 23, Department of Anthropology, University of Massachusetts at Amherst: 5-16.

Blazina, C. (2003): The Cultural Myth of Masculinity. Praeger Publishers, Westport.

Bourdieu, P. (1975): La spécificité du champ scientifique et les conditions sociales du progrès de la raison. Sociologie et Sociétés, VII (1): 91-118.

Caballero, C. (2010): Prólogo. En Arnaus y Piussi 2010: 9-15.

Cárdaba, G.; Cruz Berrocal, M.; González, C.; Mansilla, A. M.; Rodríguez de la Esperanza, M. J.; Ruiz, C.; Tormo, M. (1998): Las primeras generaciones de arqueólogas españolas: una aproximación. Revista d'Arqueologia de Ponent, 8: 151-166.

Castro, P.; Chapman, R.; Gili, S.; Lull, V.; Micó, R.; Rihuete, C.; Risch, R.; Sanahuja, M. E. (1993-4): Tiempos sociales de los contextos funerarios argáricos. Anales de Prehistoria y Arqueología, 9-10: 77-107.

Collins, P. H. (1990): Black Feminist Thought: Knowledge, Consciousness, and the Politics of Empowerment. Routledge, Nueva York.

Colomer, L. (1996): Contenidors ceràmics i processament d'aliments a la prehistòria. Cota Zero, 12: 47-60.

Colomer, L. (2005): Cerámica prehistórica y trabajo femenino en El Argar: una aproximación desde los estudios de la tecnología cerámica. Arqueología y Género (M. Sánchez Romero, ed.), Universidad de Granada, Granada: $177-217$.

Colomer, L.; González Marcén, P.; Montón Subías, S. (1998): Maintenance activities, Technological Knowledge and Consumption Patterns: A View of Northeast Iberia (2000-500 Cal BC). Journal of Mediterranean Archaeology, 11 (1): 53-80.

Colomer, L.; González Marcén, P.; Montón Subías, S.; Picazo, M. (eds.) (1999): Arqueología y teoría feminista. Estudios de mujeres y cultura material en arqueología. Icaria, Barcelona.

Colomer, E.; Gili, S.; González Marcén, P.; Montón Subías, S.; Picazo, M.; Rihuete, C.; Ruiz, M.; Sanahuja, M. E.; Sanz, T.; Tenas, M. (1992): ¿Somos todas hombres? Comunicación presentada en la sesión Arqueología de las Mujeres. Reunión de Arqueología Teórica. Santiago de Compostela.

Colomer, E.; Gili, S.; González Marcén, P.; Montón Subías, S., Picazo, M.; Rihuete, C.; Ruiz, M.; Sanahuja, M. E.; Tenas, M. (1993): Género y Arqueología: las mujeres en la prehistoria. Arqrítica 6: 5-7.

Comins, I. (2009): Filosofía del cuidar. Una propuesta coeducativa para la paz. Icaria, Barcelona.

Conkey, M.; Gero, J. (1997): Programme to Practice: Gender and Feminism in Archaeology. Annual Review of Anthropology, 26: 411-38.

Conkey, M.; Spector, J. (1984): Archaeology and the Study of Gender. Advances in Archaeological Method and Theory (M. Schiffer, ed.), Academic Press, Nueva York: 1-38.

ConKey, M. (2005): Dwelling at the margins, action at the intersection? Feminist and indigenous archaeologies. Archaeologies, 1: 9-59.

Connell, R.W. (1995): Masculinities. Allen \& Unwin, Sydney.

Connell, R.J.; Messerschmidt, J.W. (2005): Hegemonic masculinity: rethinking the concept. Gender \& Society, 19 (6): 829-59.

Cruz Berrocal, M. (2009): Feminismo, teoría y práctica de una arqueología científica. Trabajos de Prehistoria, 66 (2): 25-43.

Cruz Berrocal, M. (en prensa): Archaeology is (sometimes) History, or it is nothing. The Prehistory of Iberia. Debating Early Social Stratification and the State (M. Cruz Berrocal, L. García y A. Gilman, eds.), Routledge, Londres.

Curià, E.; PicAzo, M. (2000): Cambios del poblamiento rural en El Empordà durante la etapa de transición a la romanización. Els productes alimentaris d'origen vegetal a l'Edat del Ferro de l'Europa occidental: de la producció al consum (R. Buxó y E. Pons, eds.), Museu d'Arqueologia de Catalunya, Girona: 87-94.

Delgado, A.; Ferrer, M. (2007): Alimentos para los muertos: mujeres, rituales funerarios e identidades coloniales. Treballs d'Arqueologia, 13: 29-67. 
Díaz-Andreu, M.; Sanz, N. (1994): Women in Spanish Archaeology. Archaeological Papers of the American Anthropological Association, 5(1): 121-30.

DíAz-Andreu, M. (2005): Género y arqueología: una nueva síntesis. Arqueología y Género (M. Sánchez Romero, ed.), Granada, Universidad de Granada, Granada: 13-51.

Díaz-Andreu, M.; Montón Subías, S. (2012): Feminist and Gender Studies in Southwestern Europe: Spanish, Portuguese and French Prehistoric Archaeologies. A Companion to Gender Prehistory (D. Bolger, ed.), WileyBlackwell, Malden: 438-457.

Djerassi, C. (1989): Cantor's Dilemma. Penguin, Nueva York.

Duerassi, C. (1998): No. Penguin, Nueva York.

Dowson, T. A. (1998): Homosexualitat, teoria queer i arqueologia. Cota Zero, 14: 81-87.

Easthope, A. (1990): What a Man's Gotta Do. The Masculine Myth in Popular Culture. Routledge, Nueva York.

Engelstad, E. (1999): Imágenes de poder y contradicción: Teoría feminista y Arqueología Posprocesual. Arqueología y Teoría Feminista. Estudios Sobre Mujeres y Cultura Material en Arqueología (L. Colomer, P. González Marcén, S. Montón Subías, M. Picazo, eds.), Icaria, Barcelona: 69-96.

Engelstad, E. (2007): Much More than Gender. Journal of Archaeological Method and Theory, 14: 217-34.

EsCORIZA, T. (2002): La representación del cuerpo femenino. Mujeres y arte rupestre levantino del arco mediterráneo de la Península Ibérica. B.A.R., Oxford.

Escoriza, T.; SAnahuja, M. E. (2005): La Prehistoria de la autoridad y la relación. Nuevas perspectivas de análisis para las sociedades del pasado. Arqueología y Género (M. Sánchez Romero, ed.), Granada, Universidad de Granada, Granada: 109-140.

Fernández Martínez, V.M. (2006): Una Arqueología Crítica. Ciencia, ética y política en la construcción del pasado. Crítica, Barcelona.

Fernández-Posse, M. D. (2000): La mujer en la cultura castreña astur. Arqueología Espacial, 22: 143-160.

Franklin, M. (2001): A Black feminist-inspired archaeology? Journal of Social Archaeology, 1(1): $108-125$.

Gero, J. (1996): Archaeological practice and gendered encounters with field data. Gender and Archaeology (R. P. Wright, ed.), University of Pennsylvania Press, Philadelphia: 251-280.

Giddens, A. (1984): The constitution of society: outline of the theory of structuration. Polity Press, Cambridge.

Gilligan, C. (1982): In a different voice. Harvard University Press, Cambridge, Massachusetts.

GonzÁlez MARcÉn, P. (2006): Dones i prehistòria: viure el present, pensar el passat. Les dones en la prehistòria (B. Soler, ed.), Museu de Prehistòria, Valencia: 15-26.

GonzÁlez Marcén, P. (2008a): La otra prehistoria: creación de imágenes en la literatura científica y divulgativa. Arenal, 15: 91-109.

GonzÁlez Marcén, P. (2008b): Tiempos de mujeres. Escala de análisis y metodología arqueológica. Arqueología del Género. ler Encuentro Internacional en la UAM (L. Prados y C. Ruíz, eds.), Universidad Autónoma de Madrid, Madrid: 61-75.

GonzÁlez Marcén, P.; Picazo, M. (2005): Arqueología de la vida cotidiana. Arqueología y Género (M. Sánchez Romero, ed.), Universidad de Granada, Granada: 141-158.

González Marcén, P.; Montón Subias, S.; Picazo, M. (2008): Towards an archaeology of maintenance activities. En Montón Subías, S.; Sánchez Romero, M. 2008: 3-8.

GonzÁlez Marcén, P.; Montón Subias,S.; Picazo, M. (2007): Continuidad y cambio social en la cultura material de la vida cotidiana. Complutum, 18:175-84.

González Ruibal, A. (2011): El desastre académico de la arqueología. El futuro de la Arqueología en España (J. Almansa, ed.), JAS Arqueología, Madrid: 99-104.

GuAsch, O. (2006): Héroes, cientificos, heterosexuales y gays. Los varones en perspectiva de género. Bellaterra, Barcelona.

Harding, S. (1986): The Science Question in Feminism. Cornell University Press, Londres.

Hartsock, N. (1983): The Feminist Standpoint: developing the ground for a specifically feminist historical materialism. Discovering Reality: Feminist Perspectives in Epistemology,Metaphysics, Methodology and Philosophy of Science (S. Harding y M. Hintikka, eds.), D. Reidel, Dordecht: 283-310.

HERnÁNDEZ, L. (2005): La mujer de la prehistoria: un sujeto activo. El conocimiento del pasado. Una herramienta para la igualdad (M. C. Sevillano; J. Rodríguez; M. Olarte y L. Lahoz, eds.), Plaza Universitaria, Salamanca: 75-86. 
Hernando, A. (2000): Hombres del tiempo y mujeres del espacio: individualidad, poder y relaciones de género. Arqueología espacial, 22: 23-44.

Hernando, A. (2002): Arqueología de la identidad. Akal, Madrid.

Hernando, A. (2005): Mujeres y Prehistoria. En torno a la cuestión del origen del patriarcado. Arqueología y Género (M. Sánchez Romero, ed.), Universidad de Granada, Granada: 73-108.

Hernando, A. (2010): Gender, Individual, and Affine/Consanguineal Relationships in 'Egalitarian Societies': The Awá-Guajá Case. Situating Gender in European Archaeologies (L. H. Dommasnes; T. Hjørungdal; S. Montón Subías; M. Sánchez-Romero y N. Wicker, eds.), Archaeolingua, Budapest: 283-306.

Hernando, A., (2012): La fantasía de la individualidad. Sobre la construcción sociohistórica del sujeto moderno. Katz, Buenos Aires.

Hernando, A.; Politis, G.; González Ruibal, A.; Beserra, E. (2011): Gender, Power and Mobility among the Awá-Guajá (Maranhão, Brazil). Journal of Anthropological Research, 67: 189-211.

Hernando, A. (en prensa): Change, individuality and reason, ... or how archaeology has legitimized a patriarchal modernity. Reclaiming Archaeology. Beyond the tropes of modernity (A. González-Ruibal, ed.), Routledge, Londres.

Hornos, F.; Rísquez, C. (2005): Representación en la actualidad. Las mujeres en los museos. Arqueología y Género (M. Sánchez-Romero, ed.), Universidad de Granada, Granada: 479-90.

Inteman, K. (2010): 25 Years of Feminist Empiricism and Standpoint Theory: Where are We Now? Hypatia, 25 (4): $778-96$

IZQUIERDO, I. (2007): Arqueología de la muerte y el estudio de la sociedad: una visión desde el género en la cultura ibérica. Complutum, 18: 87-101.

Longino, H. (1990): Science as Social Knowledge: Values and Objectivity in Scientific Inquiry. Princeton University Press, Princeton.

LozAno, S. (2008): El sistema sexo-género en la Edad del Bronce egea: los frescos de Knossos y Akrotiri. Actas de las I Jornadas de Jóvenes en Investigación Arqueológica: Dialogando con la Cultura Material (Orjia, coord.), Cersa, Madrid: 351-358.

LozAno, S. (2011a): El hombre es la medida de todas las cosas. El tópico androcéntrico en arqueología. Estrat Crític, 5 (3): 18-29.

LozAno, S. (2011b): Gender thinking in the making: feminist epistemology and gender archaeology. Norwegian Archaeological Review, 44 (1): 21-39.

MacCormack, C. P.; Strathern, M. (eds.) (1980): Nature, Culture and Gender. Cambridge University Press, Nueva York.

MacCormack, C. P. (1980): Nature, culture and gender: a critique. En C.P. MacCormack y M. Strathern 1980: $1-24$.

Masvidal, C.; Picazo, M. (2005): Modelando la figura humana: reflexiones en torno a las imágenes femeninas de la Antigüedad. Quaderns Crema, Barcelona.

Montón Subías, S. (2000): Las mujeres y su espacio: una historia de los espacios sin historia. Arqueología espacial, 22: 45-59.

Montón SuBías, S. (2002): Cooking in zooarchaeology: is this issue still raw? Consuming passion and patterns of consumption (P. Miracle; N. Milner, ed.), McDonald Institute Monographs, Cambridge: 7-15.

Montón SUBÍAs, S. (2007): Interpreting archaeological continuities: an approach to transversal equality in the Argaric Bronze Age of south-east Iberia. World Archaeology, 39 (2): 256-62.

Montón SubíAs, S. (2010a): Muerte e identidad femenina en el mundo argárico. Trabajos de Prehistoria, 67: 119-137.

Montón SuBíAs, S. (2010b): Black Swans and Archaeological Interpretation. Norwegian Archaeological Review, 43: 1-11.

Montón SubíAs, S. (2010c): Maintenance Activities and the Ethics of Care. Situating Gender in European Archaeologies (L. H. Dommasnes; T. Hjørungdal; S. Montón Subías, M. Sánchez Romero y N. Wicker, eds.), Archaeolingua, Budapest: 23-33.

Montón Subías, S. (2011): Muerte y género en la prehistoria española. La arqueología funeraria desde una perspectiva de género (L. Prados, ed.). Universidad Autónoma de Madrid, Madrid: 21-37. 
Montón Subías, S. (en prensa): The Development of Feminist, Gender and Queer Archaeologies: A Spanish Perspective. Encyclopedia of Global Archaeology (C. Smith, ed.), Springer, Nueva York.

Montón Subías, S.; Meyer, W. (en prensa): Engendered archaeologies. Encyclopedia of Global Archaeology (C. Smith, ed.), Springer, Nueva York.

Montón Subías, S.; SÁnchez Romero, M. (eds.) (2008): Engendering social dynamics: the archaeology of maintenance activities. BAR, Oxford.

Moser, S. (1996): Science, stratigraphy and the deep sequence: Excavations vs survey and the question of gendered practice in archaeology. Antiquity, 70(270): 813-823.

Moser, S. (2007): On Disciplinary Culture: archaeology as fieldwork and its gendered associations. Journal of Archaeological Method and Theory, 14: 235-263.

Orozco, T. (2005): Cultura material y actitudes de género: el utillaje lítico tallado. Arqueología y Género (M. Sánchez Romero, ed.), Universidad de Granada, Granada: 245-60.

Ortner, S. (1974): Is Female to Male as Nature is to Culture? Women, Culture, and Society (M. Rosaldo y L. Lamphere, eds.), Stanford University Press, Stanford: 68-87.

Pallarés, M. (2000): Género y espacio social en arqueología. Arqueología Espacial, 22: 61-92.

Pedro, M. J. DE (2006): El grup domèstic i les activitats de manteniment en un llogaret de l'Edat del Bronze. La Lloma de Betxí (Paterna, València). Les dones en la prehistòria (B. Soler, ed.), Museu de Prehistòria, Valencia: $105-118$.

Penalva, J. (2011): Corrupción en la Universidad. El ocaso de la educación. El triunfo de la endogamia. Ciudadela Libros, Madrid.

Peñasco, R. (2005): Mobbing en la universidad. Adhara, Madrid.

PÉrez, M. (2011): Redescubriendo la realidad material: recuperando el feminismo materialista para la Arqueología Social. Revista Atlántica-Mediterránea de Prehistoria y Arqueología Social, 13: 47-64.

PicAzo, M. (1997): Hearth and home: the timing of maintenance activities. Invisible people and processes. Writing Gender and Childhood into European Archaeology (J. Moore y E. Scott, eds.), Leicester University Press, Londres: 59-67.

PIÑuel, I. (2005): El acoso psicológico en la Universidad. El silencio de los corderos. Prólogo a Mobbing en la universidad (R. Peñasco), Adhara, Madrid: 25-33.

Pleck, J. H. (1983): The Myth of masculinity. The MIT Press, Massachusetts.

Politis, G. (2001): On archaeological praxis, gender bias and indigenous peoples in South America. Journal of Social Archaeology, 1(1): 90-107.

Potter, E. (2006): Feminism and Philosophy of Science. An introduction. Routledge, Londres.

Prados, L. (2007): Mujer y espacio sagrado: haciendo visibles a las mujeres en los lugares de culto de época ibérica. Complutum, 18: 217-225.

Prados, L. (2010): Gender and Identity in Iberian Funerary Contexts (5th-3rd century BC). Situating Gender in European Archaeologies (L. H. Dommasnes; T. Hjørungdal; S. Montón Subías; M. Sánchez-Romero y N. Wicker, eds.), Archaeolingua, Budapest: 205-24.

Querol, M. A. (2000): El lenguaje utilizado en el tema del origen de la humanidad: una visión feminista. Primeras Jornadas Internacionales sobre Roles Sexuales y de Género. Mujer, ideología y población, Madrid: 221-238.

Querol, M. A. (2008): La imagen de la mujer en las reconstrucciones actuales de la prehistoria. Arqueología del Género. 1er Encuentro Internacional en la UAM (L. Prados y C. Ruíz, eds.), Universidad Autónoma de Madrid, Madrid: 27-42.

Querol, M. A.; Hornos, F. (2011): La representación de las mujeres en los modernos museos arqueológicos: estudio de cinco casos. Revista Atlántica-Mediterránea de Prehistoria y Arqueología social, 13: 135-156.

REeser, T. (2010): Masculinities in Theory. Wylie-Blackwell, Malden, MA.

Renfrew, C.; Bahn, P. (2012): Arqueología. Teorías, métodos y prácticas. Akal, Madrid.

Rísquez, C.; García Luque, A. (2007): ¿Actividades de mantenimiento en el registro funerario? El caso de las necrópolis íberas. Treballs d'arqueologia, 13: 145-170.

Rísquez, C.; García Luque, A.; Hornos, F. (2010): Mujeres y mundo funerario en las necrópolis ibéricas. La Dama de Baza. Un viaje femenino al más allá (T. Chapa y I. Izquierdo, eds.), Ministerio de Cultura, Madrid: 259-77.

Rivera, M. (1994): Nombrar el mundo en femenino. Pensamiento de las mujeres y teoría feminista. Icaria, Barcelona. 
Rivera, M. (2000): Una cuestión de oído. De la historia de la estética de la diferencia sexual. De dos en dos. Las prácticas de creación y recreación de la vida y la convivencia humana (M. Beltrán; C. Caballero; M. Cabré; M. Rivera y A. Vargas, eds.), Horas y Horas, Madrid: 103-122.

Rivera, M. (2005): La historia de las mujeres que nombra el mundo en femenino. Acta Historica et Archaeologica Mediaevalia, 26: 1155-1164.

Rivera, M. (2010): El Centro de Investigación Duoda ¿es la universidad? En Arnaus y Piussi 2010: 51-70.

Rose, H. (1983): Hand, Brain, and Heart: A Feminist Epistemology for the Natural Sciences. Signs: Journal of Women in Culture and Society, 9(1): 73-90.

Ruiz, A. (2008): Eulalia Guzmán y la imposibilidad de excavar en suelo nacional. Cuiculco, 15 (43): $137-157$.

SAlAZAR, O. (2012): Otras masculinidades posibles: Hacia una humanidad diferente y diferenciada. Recerca, 12: 87-112.

Sanahuja, M.E.; Picazo, M. (1989): Los estudios de las mujeres a lo largo de la Prehistoria y en la Antigüedad griega: estado de la cuestión. Arqrítica 1: 32-37.

Sanahuja, M. E. (1991): Modelos explicativos sobre los orígenes y evolución de la humanidad. Mujeres y sociedad. Nuevos enfoques teóricos y metodológicos (L. Luna, coomp.), Universidad de Barcelona, Barcelona: 149-166.

Sanahuja, M. E. (2002): Cuerpos sexuados, objetos y prehistoria. Feminismos-Cátedra, Barcelona.

Sanahuja, M. E. (2006): Dones, homes i aixovars funeraris. Les dones en la prehistòria (B. Soler, ed.), Museu de Prehistòria, Valencia: 79-89.

SÁnchez Liranzo, O. (2008): El debate teórico en los estudios de la arqueología del género y su incidencia en la prehistoria. Arqueología del Género. ler Encuentro Internacional en la UAM (L. Prados y C. Ruíz, eds.), Universidad Autónoma de Madrid, Madrid: 43-60.

SÁnchez Romero, M. (2000): Mujeres y espacios de trabajo en el yacimiento de los Castillejos (Montefrío). Arqueología Espacial, 22: 93-106.

SÁnchez Romero, M. (2007): Actividades de mantenimiento en la edad del bronce del sur peninsular: el cuidado y la socialización de individuos infantiles. Complutum, 18: 25-34.

SÁnchez Romero, M. (2008): An approach to learning and socialization in children during the Spanish Bronze Age. Children Identity and the past (L. H. Dommasnes y M. Wrigglesworth, eds.), Cambridge Scholars Publishing, Cambridge: 113-124.

SÁnchez Romero, M. (2009): La arqueología de las mujeres y las relaciones de género en España: una revisión bibliográfica. Los estudios de las mujeres de España y Argentina: propuesta para el debate (M. E. Fernández; N. Bonaccorsi y C. Lagunas, eds.), Prometeo Libros, Buenos Aires: 79-102.

SÁnchez Romero, M.; Aranda, G. (2005): El cambio en las actividades de mantenimiento durante la Edad del Bronce: nuevas formas de preparación, presentación y consumo de alimentos. Treballs d'Arqueologia, 11: 73-90.

SÁnchez Romero, M.; Moreno, A. (2005): Mujeres y producción metalúrgica en la prehistoria: el caso de Peñalosa (Baños de La Encina, Jaén). Arqueología y Género (M. Sánchez Romero, ed.), Universidad de Granada, Granada: 261-81.

SEIDler, V. (2000): La sinrazón masculina. Masculinidad y teoría social. UNAM, Centro Superior de Investigaciones y Estudios Superiores en Antropología Social, Paidós Mexicana, México D.F.

Shiva, V. (1998): Las mujeres en la naturaleza. Ecología y feminismo (Agra Romero, M. X., ed.), Comares, Granada: $161-177$

Simone, R. De (2000): L'università dei tre tradimenti. Laterza, Roma.

Sмiтh, D. (1988): The Everyday World as Problematic: a feminist sociology. Northeastern University Press, Boston. Soler, B. (2008): De la investigación a la difusión: el museo como vehículo de mediación. Arenal, 15: $174-194$. TAnesini, A. (1999): An Introduction to Feminist Epistemologies. Blackwell, Oxford.

TomÁšKovÁ, S. (2007): Mapping a Future: Archaeology, Feminism and Scientific Practice. Journal of Archaeological Method and Theory, 14: 264-284.

Vargas, I. (2004): Hacia una Teoría Feminista en Arqueología. Otras Miradas, 4(2): 62-75.

VAzquez-Cupeiro, S.; Elston, M. (2006): Gender and academic career trajectories in Spain. From gendered passion to consecration in a 'Sistema Endogámico'? Employee Relations, 28 (6): 588-603. 
Vicent, J. (1994): Perspectivas de la Teoría Arqueológica en España. Sexto Coloquio Hispano-Ruso de Historia, Fundación Cultural Banesto, Madrid: 215-22.

Vila, A. (2011): Política y feminismo en arqueología prehistórica. Revista Atlántica- Mediterránea de Prehistoria y Arqueología social, 13: 17-32.

ViTA, A. DE (2010): En la universidad: calidad de vida y vida asociada. En Arnaus y Piusi 2010: 79-90.

Voss, B. (2008): Sexuality Studies in Archaeology. Annual Review of Anthropology 37: 317-336.

Watkins, J. (2000): Indigenous Archaeology. American Indian Values and Scientific Practice. Altamira Press, Walnut Creek.

Wylie, A. (2003): Why Standpoint Matters. Philosophical Explorations of Science, Technology and Diversity ( R. Figeroa y S. Harding, eds.) Routledge, Londres y Nueva York: 26-49.

Wylie, A. (2007): Doing Archaeology as a Feminist: An Introduction. Journal of Archaeological Method and Theory, 14: 209-216.

Zamboni, C. (2010): El juego de las damas y el ajedrez. En Arnaus y Piusi 2010: 71-78.

Zarzalejos, M. M. (2008): Los estudios de arqueología del género en la Hispania Romana. Arqueología del Género. ler Encuentro Internacional en la UAM (L. Prados y C. Ruíz, eds.), Universidad Autónoma de Madrid, Madrid: 297-326. 